\title{
Broadband infrared generation with noncollinear optical parametric processes on periodically poled $\mathrm{LiNbO}_{3}$
}

\author{
Chih-Wei Hsu and C. C. Yang \\ Department of Electrical Engineering and Graduate Institute of Electro-Optical Engineering, National Taiwan University, \\ 1, Roosevelt Road, Section 4, Taipei, Taiwan \\ Received April 9, 2001 \\ Broadband signal and idler generation based on the spectral retracing behavior in noncollinear phase matching \\ of optical parametric generation in periodically poled $\mathrm{LiNbO}_{3}$ (PPLN) is reported. Using PPLN of 29.5- $\mu \mathrm{m}$ \\ quasi-phase-matching period and a $Q$-switched Nd:YAG laser as a pump, we obtained a broad signal spec- \\ trum from 1.66 to $1.96 \mu \mathrm{m}$ and corresponding idler wavelengths from 2.328 to $2.963 \mu \mathrm{m}$. The experimental \\ results were consistent with theoretical predictions. Circular and elliptical pump beams were also compared. \\ (C) 2001 Optical Society of America \\ OCIS codes: $190.0190,190.4970$.
}

Broadband optical sources can find applications in many areas, such as wavelength-division multiplexing (WDM) signal generation and amplification of fiber communications, optical coherence tomography, and spectroscopy with large excitation tunability. For WDM communications, besides conventional erbium-doped fiber amplification and stimulated Raman scattering in fiber, $\chi^{(2)}$ nonlinear optics has been considered for broadband signal amplification based on engineered quasi-phase matching (QPM) in $\mathrm{LiNbO}_{3} .{ }^{1}$ In the application of optical coherence tomography, a broader source bandwidth results in a higher spatial resolution in the scanning-depth dimension. ${ }^{2}$ Although light generation based on $\chi^{(2)}$ nonlinear optics has not been used for this purpose, its potential deserves further exploration.

Although broadband generation based on $\chi^{(2)}$ nonlinear optics can be implemented near the degenerate point in optical parametric processes, ${ }^{3,4}$ its applicable range is limited. In particular, the signal and idler contributions to broadband output usually propagate in different directions, leading to difficulty in application. By use of retracing behaviors (i.e., tuning of the phase-matching curve) of the birefringence phase-matching angle in various nonlinear crystals (either collinear or noncollinear), broadband signal generation of more-applicable ranges has been obtained. $^{5-8}$ A $\beta$-barium borate optical parametric oscillator pumped with the second harmonic of a $Q$-switched Nd:YAG laser has produced a signal bandwidth from 745 to $890 \mathrm{~nm}$, with a corresponding idler wavelength from 1.32 to $1.86 \mu \mathrm{m} .^{8}$ Although the QPM method in the collinear configuration can increase the interaction length without the walk-off problem's occurring, some reports have demonstrated the advantages of angle-tuned optical parametric oscillator with periodically poled $\mathrm{LiNbO}_{3}$ (PPLN; Refs. 9 and 10) and KTP (Ref. 11) based on noncollinear interaction. In this Letter we report the implementation of broadband optical parametric generation (OPG) in PPLN, based on a novel phase-matching retracing behavior. The retracing behavior was implemented with noncollinear pumping of a PPLN optical para- metric process. Although noncollinear pumping of QPM nonlinear processes has been reported, ${ }^{9-11}$ retracing behaviors for broadband generation such as those in our results have not, to our knowledge, been discussed in the literature. Here we show the experimental and theoretical results of broadband OPG in PPLN pumped with a $Q$-switched Nd:YAG laser. The signal wavelength can be as broad as $300 \mathrm{~nm}$, with the central wavelength ranging from 1.66 to $1.96 \mu \mathrm{m}$. Results of pump-angle variation and temperature tuning are also presented. In addition, the differences between circular and elliptical pump beams are discussed. Recently, broadband generation in PPLN, either operated near the degenerate point or based on a well-designed fan QPM structure, was reported. ${ }^{12}$ However, a retracing mechanism was not utilized or discussed.

Figure 1 shows the noncollinear phase-matching geometry. $\theta$ is defined as the angle between the pump-beam direction and the normal to the QPM grating inside PPLN. The angle between pump and signal beams is $\delta$, and the angle between the pump and idler beams is $\gamma . \quad \alpha$ represents the incident angle

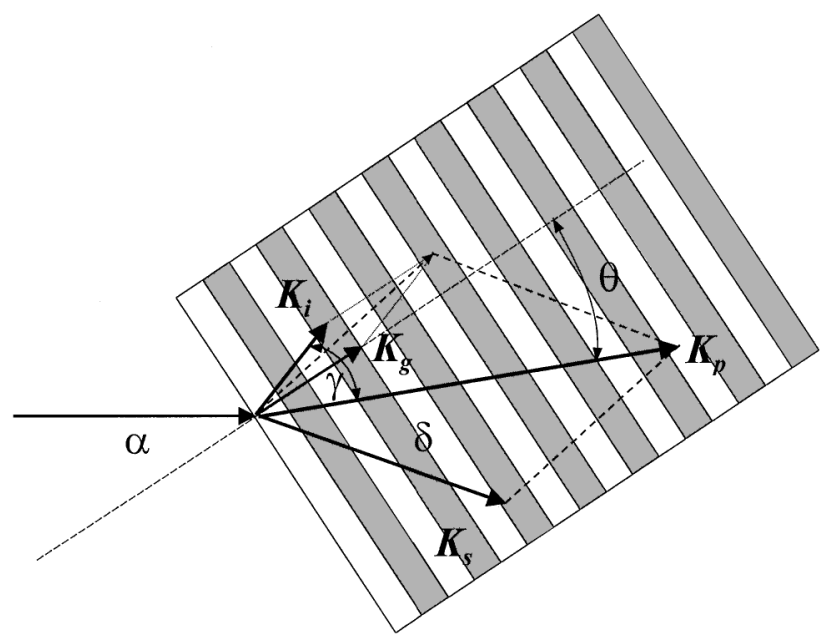

Fig. 1. Definitions of various wave vectors and angles in the noncollinear phase-matching configuration. 
of the pump in air. The phase-matched angles for the signal and idler are described as follows:

$$
\begin{aligned}
K_{i} \cos \gamma+K_{g} \cos \theta+K_{s} \cos \delta & =K_{p}, \\
K_{i} \sin \gamma+K_{g} \sin \theta & =K_{p} \sin \delta .
\end{aligned}
$$

Here, $K_{p}, K_{s}, K_{i}$, and $K_{g}$ represent the magnitudes of the wave vectors of the pump $\left(\mathbf{K}_{p}\right)$, signal $\left(\mathbf{K}_{s}\right)$, idler $\left(\mathbf{K}_{i}\right)$, and QPM grating $\left(\mathbf{K}_{g}\right)$, respectively. The signal is defined to have a wavelength shorter than that of the idler. Figure 2(a) shows the theoretical predictions of the phase-matching condition for OPG. The signal wavelength is shown as a function of pump angle $\theta$ based on 29.5- $\mu \mathrm{m}$-period PPLN with temperature varying from 160 to $200{ }^{\circ} \mathrm{C}$. We assume that the idler propagates along the normal to the QPM structure $(\gamma=\theta)$. The pump wavelength is $1.064 \mu \mathrm{m}$. Retracing behavior of the phase-matching angle can be clearly seen when the signal wavelength is near $1.8 \mu \mathrm{m}$. Such retracing behavior can result in broadband generation. Based on our calculations, similar phenomena can be observed with other pump wavelengths and periods of PPLN. Figure 2(b) shows the retracing behaviors of phase-matching angle $\theta$ with a PPLN period of $\sim 24 \mu \mathrm{m}$ when the pump wavelength is $900 \mathrm{~nm}$ and the temperature is fixed at $179^{\circ} \mathrm{C}$. It is assumed that the signal propagates along the normal to the QPM structure. One can see that the phase-matching curve becomes almost vertical near $\theta=1.252^{\circ}$ when the PPLN period is $24.3 \mu \mathrm{m}$. We predict that, with appropriate experimental conditions, a broadband source ranging from 1.42 to $1.65 \mu \mathrm{m}$ will be feasible. Such an implementation will be useful for WDM fiber communications.

In our experiment, a $Q$-switched Nd:YAG laser (Coherent Infinity 40-100) with a pulse width of $3.5 \mathrm{~ns}$ was used for pumping. We employed a lens system to focus the pump beam to a waist of $190-\mu \mathrm{m}$ diameter inside a PPLN crystal. The 1.9 -cm-long PPLN crystal contained eight different QPM-period structures. Each structure was $1.3 \mathrm{~mm}$ in width and separated by $\sim 0.1 \mathrm{~mm}$ from the neighboring structures. The pump-beam coverage of the other QPM periods was small. The crystal was placed in an oven whose temperature could be varied up to $250{ }^{\circ} \mathrm{C}$ with an accuracy of $0.1^{\circ} \mathrm{C}$.

Figure 3 shows four signal spectra of different pump angles with $29.5-\mu \mathrm{m}$-period PPLN and the temperature fixed at $190^{\circ} \mathrm{C}$. When we rotated the PPLN crystal to increase $\alpha$ (and hence $\theta$ ), the central wavelength $\left(\lambda_{\mathrm{sc}}\right)$ and the FWHM spectral width $\left(\Delta \lambda_{s}\right)$ of the signal spectra varied from $\lambda_{\mathrm{sc}}=1.563 \mu \mathrm{m}$ with $\Delta \lambda_{s}=8.5 \mathrm{~nm}$ (curve I, the collinear case) to the broadest case of $\lambda_{\mathrm{sc}}=1.81 \mu \mathrm{m}$ with $\Delta \lambda_{s}=300 \mathrm{~nm}$ (curve III, from 1.66 to $1.96 \mu \mathrm{m}$ at $\alpha=4^{\circ}$; the corresponding value of $\theta$ is $1.85^{\circ}$. Because of water-vapor absorption near $2 \mu \mathrm{m}$ and bandwidth limitation of our spectrum analyzer, the broadest signal spectrum is nonsymmetric. With a further increase of $\theta$ to $2.3^{\circ}$, the FWHM spectral width was reduced to $140 \mathrm{~nm}$ (curve IV, centered at $1.84 \mu \mathrm{m}$ ). In the case of the broadest signal spectral width, the corresponding bandwidth of the idler is
$635 \mathrm{~nm}$ near $2.5 \mu \mathrm{m}$. In these experiments the pump threshold for observing broadband output with our detection facilities increased from 0.334 to $1.25 \mathrm{~mJ}$ as $\theta$ varied from 0 to $2.3^{\circ}$. Such a threshold increase is due mainly to the decreasing beam overlap as $\theta$ increases.

These results are summarized in Fig. 4. The various dashed curves represent theoretical predictions of different phase-matching conditions. The symbols stand for the signal spectral peak position, and the vertical bars show the corresponding FWHM spectral ranges. The experimental results are quite consistent with the theoretical predictions, particularly when $\theta$ is small. When $\theta$ becomes large, the experimental results differ somewhat from predictions. This difference is due to our assumption in the theoretical calculation that the idler propagates in the direction normal to the QPM grating. When $\gamma$ is large, since

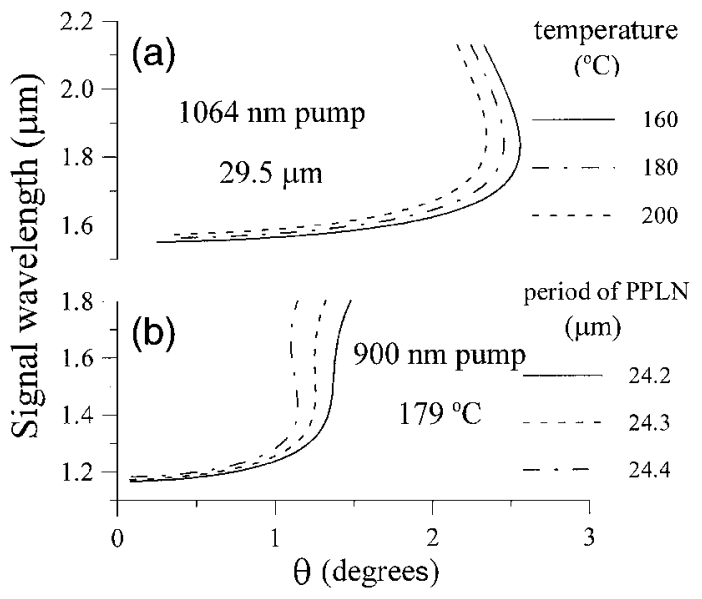

Fig. 2. (a) Theoretical angle-tuning curves with a PPLN period of $29.5 \mu \mathrm{m}$ and a pump wavelength of $1064 \mathrm{~nm}$ at several crystal temperatures. Retracing of phase-matching curves near the $1.8-\mu \mathrm{m}$ signal wavelength can be seen. (b) Theoretical tuning curve pumped with $900 \mathrm{~nm}$ at $179{ }^{\circ} \mathrm{C}$ for several PPLN periods.

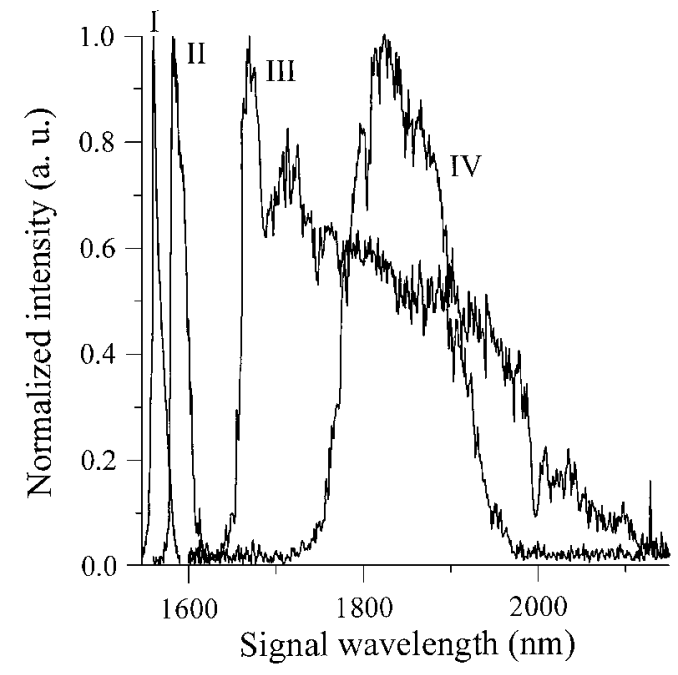

Fig. 3. Output signal spectra for several $\theta$ angles. The PPLN period is $29.5 \mu \mathrm{m}$, and the temperature is fixed at $190^{\circ} \mathrm{C}$. I, $\theta=0^{\circ}$; II, $\theta=0.92^{\circ}$; III, $\theta=1.85^{\circ}$, and $\mathrm{IV}, \theta=2.3^{\circ}$. 


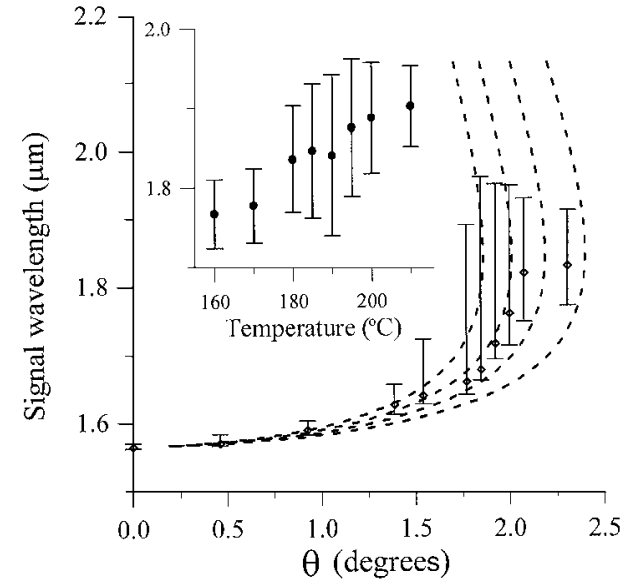

Fig. 4. Signal spectral ranges of various $\theta$ angles. The symbols indicate the spectral peak positions, and the vertical bars show the corresponding FWHM phase-matching bandwidths. The dashed curves indicate theoretical predictions for several conditions (from right to left, $\gamma=\theta$, $\gamma=1.1 \theta, \gamma=1.2 \theta, \gamma=1.3 \theta)$. The inset shows the signal spectral ranges for various crystal temperatures. The PPLN period is $29.7 \mu \mathrm{m}$. The crystal was rotated $4^{\circ}$ from its position in the collinear case.

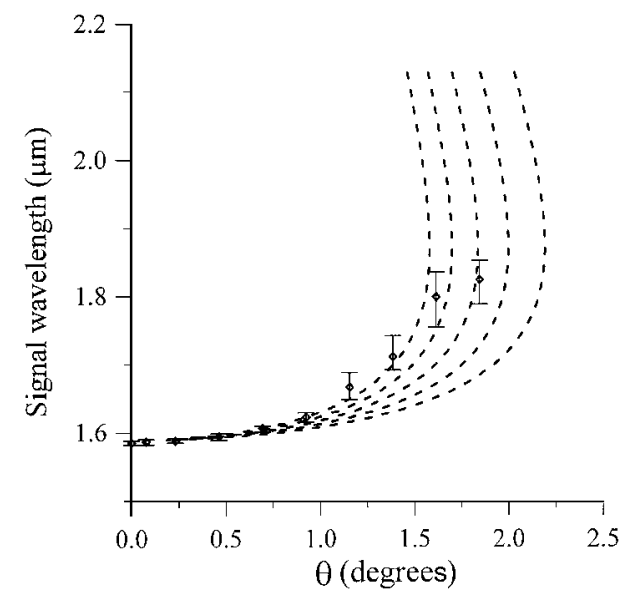

Fig. 5. Similar to Fig. 4, except that the crystal is pumped with an elliptical beam of $100 \mu \mathrm{m} \times 1 \mathrm{~mm}$. The dashed curves indicate theoretical predictions of several conditions (from right to left, $\gamma=\theta, \gamma=1.1 \theta, \gamma=1.2 \theta, \gamma=1.3 \theta$, $\gamma=1.4 \theta)$.

there is no cavity to confine the idler propagation direction, the idler may propagate in other directions. Hence the experimental bandwidth is broader than predicted. Note that different signal and idler output wavelength components propagate in different directions. Such angle chirp may lead to difficulty in collecting broadband output. However, it represents an advantage in WDM communication application.

The inset of Fig. 4 shows the temperature dependence of signal wavelength with $\alpha=4^{\circ}$. The PPLN period is now $29.7 \mu \mathrm{m}$. One can see that the acceptance temperature range for broadband generation can be several degrees centigrade. However, temperature variation leads to a change in conversion efficiency. In our experiments, we also observed efficient sumfrequency generation from the mixing of pump and sig- nals in certain directions between $\mathbf{K}_{p}$ and $\mathbf{K}_{s}$. Based on our calculations, the sum-frequency generation was due to the interaction of the noncollinear configuration with the third-order QPM.

Similarly to Fig. 4, Fig. 5 shows the results when OPG was pumped with an elliptical laser beam of $100 \mu \mathrm{m} \times 900 \mu \mathrm{m}$. The larger beam width was along the PPLN crystal surface. The PPLN period was $29.7 \mu \mathrm{m}$. Unlike in the case of a circular pump beam, the output bandwidths of the elliptical pump beam are smaller. This is so because the focused circular beam has a broader variation of the pump wave vector, leading to broader phase-matching conditions and hence broader output bandwidths. In this experiment, the pump threshold increased from $0.751 \mathrm{~mJ}$ for the collinear case to $1.15 \mathrm{~mJ}$ for $\theta=1.85^{\circ}$.

In summary, with noncollinear OPG on PPLN, we have theoretically predicted retracing behaviors of phase matching and experimentally demonstrated the generation of broadband signal and idler beams. The broadest signal spectrum covered the wavelength range from 1.66 to $1.96 \mu \mathrm{m}$. The corresponding idler wavelength ranged from 2.328 to $2.963 \mu \mathrm{m}$. Note that the OPG output bandwidth reported can be reduced if an OPO is implemented because of the propagation direction confinement of either the signal or the idler. Such a reduction will not affect broadband generation much if the case shown in Fig. 2(b) is considered.

This research was supported by the National Science Council of the Republic of China under grants NSC 89-2218-E-002-094, NSC 89-2218-E-002-095, and NSC 89-2215-E-002-051. C. C. Yang's e-mail address is ccy@cc.ee.ntu.edu.tw.

\section{References}

1. A. Galvanauskas, K. K. Wong, K. El Hadi, M. Hofer, M. E. Fermann, D. Harter, M. H. Chou, and M. M. Fejer, Electron. Lett. 35, 731 (1999).

2. W. Drexler, U. Morgner, F. X. Kartner, C. Pitris, S. A. Boppart, X. D. Li, E. P. Ippen, and J. G. Fujimoto, Opt. Lett. 24, 1221 (1999).

3. A. J. Campillo, R. C. Hyer, and S. L. Shapiro, Opt. Lett. 4, 357 (1979).

4. A. Birmontas, A. Piskarskas, and A. Stabinis, Sov. J. Quantum Electron. 13, 1243 (1983).

5. S. Lin, B. Wu, F. Xie, and C. Chen, Appl. Phys. Lett. 59, 1541 (1991).

6. X. Liu, D. Deng, M. Li, D. Guo, and Z. Xu, J. Appl. Phys. 74, 2989 (1993).

7. J. Wang, M. H. Dunn, and C. F. Rae, Opt. Lett. 22, 763 (1997).

8. S. D. Huang, C. W. Hsu, D. W. Huang, and C. C. Yang, J. Opt. Soc. Am. B 15, 1375 (1998).

9. S. T. Yang and S. P. Velsko, Opt. Lett. 24, 133 (1999).

10. M. J. Missey, V. Dominic, P. E. Powers, and K. L. Schepler, Opt. Lett. 24, 1227 (1999).

11. V. Smilgevicius, A. Stabinis, A. Piskarskas, V. Pasiskevicius, J. Hellstrom, S. Wang, and F. Laurell, Opt. Commun. 173, 365 (2000).

12. S. M. Russell, M. J. Missey, P. E. Powers, and K. L. Schepler, in Conference on Lasers and Electro-Optics (CLEO), Vol. 56 of OSA Trends in Optics and Photonics Series (Optical Society of America, Washington, D.C., 2001), paper CThJ4. 